\title{
ESTUDI0 GENERAL
}

\section{SITUACIÓN DE LA CEPA EPIDÉMICA DE NEISSERIA MENINGITIDIS C:2b:P1.2,5 DESPUÉS DE LA VACUNACIÓN A+C}

\author{
Belén Alcalá Galicia y Julio A. Vázquez Moreno \\ Laboratorio de Referencia de Meningococos. Centro Nacional de Microbiología. Instituto de Sálud Carlos III. \\ Ministerio de Sanidad y Consumo
}

\section{RESUMEN}

Tras la utilización de una amplia campaña de inmunización en la población de entre 2 y 19 años de edad en la mayoría de las Comunidades Autónomas, con objeto de controlar el «brote u onda epidémica» por serogrupo $\mathrm{C}$ de meningococos de los años 1996-97, interesaba conocer la situación de la conocida cepa epidémica C:2b:P1.2,5, que había sido identificada como la causante de los cambios producidos en el perfil epidemiológico de la enfermedad meningocócica en España, lo que nos podría permitir analizar algunas de las posibles tendencias futuras de esta enfermedad en nuestro país. El análisis de la situación en los primeros 10 meses de 1999 revela que se ha vuelto a cifras semejantes a las que se daban, tanto en numero de casos como en frecuencia de serogrupos, similar a la que se observaba en España a principios de los años 90, aunque la cepa epidémica C:2b:P1.2,5 representa el $56 \%$ del total de casos de serogrupo C. Las cepas de serotipo 2a, muy frecuentes en el serogrupo $C$ en países Europeos, siguen representando una minoría en España. Es interesante finalmente mencionar la aparición de variantes antigénicas que podrían ser resultado de procesos de recombinación genética intraespecíficos y que serían seleccionados en función de sus ventajas evolutivas.
ABSTRACT

\section{Situation of the Epidemic Strain of \\ Neisseria Meningitidis c:2b:P1.2,5 Following A+C Vaccination}

Following the use of an extensive immunisation campaign targeting the population between ages 2 and 19 in the majority of the Autonomous Communities (Regions), for the purpose of controlling the "outbreak or epidemic wave» caused by serogroup C in 1996-97, there was great interest in ascertaining the situation of the well-known epidemic strain C:2b:P1.2.5, which had been identified as the cause of the changes brought about in the epidemiological profile of meningococcal disease in Spain, as this would enable us to analyse some of the possible future tendencies of this disease in our country. An analysis of the situation in the first 10 months of 1999 reveals that we have reverted to figures similar to those which, both insofar as the number of cases as well as the frequency of serogroups, were observed in Spain at the beginning of the decade of the nineties, although the epidemic strain C:2b:Pl. 2.5 represents $56 \%$ of the total cases of serogroup $C$. The strains of serotype $2 \mathrm{a}$, very frequent in serogroup $\mathrm{C}$ in European countries, continue to represent a minority in Spain. Finally, it is of interest to mention the appearance of antigen variants which could be the result of processes of intra-specific genetic recombination and which would presumably have been selected in terms of their evolutive advantages.

\section{COMUNICACIÓN}

En los últimos 10 años hemos asistido en España a una más que interesante y a la vez

Correspondencia:

Julio A. Vázquez Moreno

Laboratorio de Referencia de Meningococos

Centro Nacional de Microbiología

Instituto de Salud Carlos IIl

Majadahonda

28220 Madrid cambiante situación epidemiológica en el perfil de la enfermedad meningocócica, inicialmente provocada por la aparición de una nueva cepa epidémica, caracterizada como C:2b:P1.2,5, y que provocó un cambio en el serogrupo predominante, con un aumento asociado en el número de casos, dando lugar al conocido «brote u onda epidémica» por serogrupo $\mathrm{C}$ de los años 1996 y $1997^{1}$. Así, el porcentaje de cepas con serogrupo $\mathrm{C}$ recibidas en el Laboratorio de $\mathrm{Re}$ ferencia de Meningococos pasó de estar alrede- 
dor del $30 \%$ hasta 1994 , ascendiendo al $42 \%$ en 1995 y pasando a un $60 \%$ en los años mencionados. Sin embargo, la utilización del recurso de una campaña de inmunización en la población de entre 2 y 19 años de edad en la mayoría de las Comunidades Autónomas, provocó un nuevo cambio, volviendo a bajar el porcentaje del serogrupo $\mathrm{C}$ a unos niveles semejantes a los registrados en 1995 , en torno al $40 \%$. La mayoría de las Comunidades que optaron por la campaña de vacunación, presentaban en los primeros 10 meses de 1999 entre un $20 \%$ y un $30 \%$ de casos por serogrupo $\mathrm{C}$. Cuatro Comunidades mostraban una frecuencia de casos por cepas de serogrupo $\mathrm{C}$ superior al 50\%: Andalucía, Navarra, Canarias y Baleares, si bien en estas dos últimas hay un elevado número de casos que, por características epidemiológicas y microbiológicas, deben ser considerados como importados, con lo que los datos las situarían en ese momento en parecidos niveles a los observados en el resto.

Con esta nueva realidad, era muy interesante conocer la situación de la conocida cepa epidémica $\mathrm{C}: 2 \mathrm{~b}: \mathrm{P} 1.2,5$, que había sido identificada como la causante del brote epidémico ${ }^{2}$, lo que nos podría permitir analizar algunas de las posibles tendencias futuras de la enfermedad meningócica en España. En este punto debemos recordar que en los años anteriores a 1994, la cepa C:2b:P1.2,5 sólo tenía una presencia testimonial, representando menos del $2 \%$ de las cepas de serogrupo C. Este porcentaje fue posteriormente ascendiendo, llegando a ser de un $70 \%$ durante 1996 y 1997. La caída posterior en el número de casos de enfermedad meningocócica no ha conducido a una situación semejante a la que se daba a principios de la década de los años 90 , y durante los 10 primeros meses de 1999, la cepa epidémica aún representó un $56 \%$ del total de meningococos de serogrupo $\mathrm{C}$. Si bien las cepas de meningococo de serotipo $2 b$ son aisladas en otros países europeos ${ }^{3}$, las cepas de serotipo 2 a son mayoría, constituyendo el caso español una excepción. Esta situación de mayoría de cepas del serotipo $2 b$ frente a las $2 \mathrm{a}$ se invierte en las islas, tanto en Canarias como especialmente en Baleares, donde las cepas de serotipo 2a son cerca del $50 \%$ en el primer caso, y más del $80 \%$ en el segundo, a causa del alto número de turismo europeo, y especialmente del Reino Unido, que ambas Comunidades reciben. En este sentido, el Reino Unido ha sufrido un aumento en el número de casos de enfermedad meningocócica por serogrupo $\mathrm{C}$, especialmente de serotipo $2 \mathrm{a}$, que le ha llevado a realizar una campaña de vacunación con una nueva vacuna conjugada frente a meningitis meningócica de serogrupo $\mathrm{C}$, que parece ser eficaz a partir de los 2 meses de edad, produciendo además una protección de mayor duración que la que se conseguía con la vacuna de polisacáridos, especialmente en niños de corta edad.

Por último, es muy importante recordar que en todo análisis moderno de situaciones epidemiológicas provocadas por procesos microbianos es de enorme interés el análisis de los posibles eventos de recombinación genética que puedan estar produciéndose en el periodo de tiempo analizado. Estos procesos van a dar lugar a variantes genéticas que expresen diversas modalidades antigénicas que vienen a modificar la situación analizada. Neisseria meningitidis ha sido definido como un microorganismo altamente transformable, en el que es relativamente frecuente la aparición de este tipo de variantes antigénicas, habiéndose descrito mecanismos de «switch on-off» en diversos genes, y particularmente en los genes que codifican para la cápsula ${ }^{4}$. En nuestro laboratorio hemos recibido algunas cepas, aún en número de escasa relevancia, que podrían ser cepas C:2b:P1.2,5 que por procesos de recombinación estarían ahora expresando una cápsula de serogrupo $\mathrm{B}$, siendo pues caracterizadas como B:2b:P1.2,5, lo que les ofrece una ventaja evolutiva para evadir la respuesta inmune generada por inmunización.

\section{BIBLIOGRAFÍA}

1. Mateo S, Cano R, García C. Changing epidemiology of meningococcal disease in Spain, 1989-1997. Eur Communicable Dis Bull 1997; 2(10): 71-74.

2. Berrón S, De La Fuente L, Martín E, Vázquez JA. Rising incidence of meningococcal disease in Spain associated with a new variant of serogroup C. Eur J Clin Microbiol Infect Dis, 1998; 17: 85-89. 
3. Connolly $\mathrm{M}, \mathrm{Noah} \mathrm{N}$. Is group $\mathrm{C}$ meningococcal disease increasing in Europe? A report of surveillance of meningococcal infection in Europe 1993-6. European Meningitis Surveillance

Group. Epidcmiol Infect 1999 Fcb;122 (1): $41-9$
4. Swartley JA, Marfin AA, Edupuganti S, Liu LJ, Cieslak P, Perkins B, Wenger JD, Stephens DS. Capsule switching of Neisseria meningitidis. Proc Natl Acad Sci USA, 1997; 94: 271-276. 